\title{
Plasmodium vivax
}

National Cancer Institute

\section{Source}

National Cancer Institute. Plasmodium vivax. NCI Thesaurus. Code C123550.

A protozoan parasite in the family Plasmodiidae. P. vivax is the most frequent and widely distributed causative agent of tertian malaria. 